\title{
MATHEMATICAL MODELLING FOR CORONAVIRUS DISEASE (COVID-19) IN PREDICTING FUTURE BEHAVIOURS AND SENSITIVITY ANALYSIS
}

\author{
Sarbaz H. A. Khoshnaw ${ }^{1, *}$, Rizgar H. Salih ${ }^{1}$ \\ AND SADEGH SulaimanY²
}

\begin{abstract}
Nowadays, there are a variety of descriptive studies of available clinical data for coronavirus disease (COVID-19). Mathematical modelling and computational simulations are effective tools that help global efforts to estimate key transmission parameters. The model equations often require computational tools and dynamical analysis that play an important role in controlling the disease. This work reviews some models for coronavirus first, that can address important questions about the global health care and suggest important notes. Then, we model the disease as a system of differential equations. We develop previous models for the coronavirus, some key computational simulations and sensitivity analysis are added. Accordingly, the local sensitivities for each model state with respect to the model parameters are computed using three different techniques: non-normalizations, half normalizations and full normalizations. Results based on sensitivity analysis show that almost all model parameters may have role on spreading this virus among susceptible, exposed and quarantined susceptible people. More specifically, communicate rate person-to-person, quarantined exposed rate and transition rate of exposed individuals have an effective role in spreading this disease. One possible solution suggests that healthcare programs should pay more attention to intervention strategies, and people need to self-quarantine that can effectively reduce the disease.
\end{abstract}

Mathematics Subject Classification. 92D30,92D25,92C42, 34C60.

Received March 30, 2020. Accepted May 12, 2020.

\section{INTRODUCTION}

Understanding and predicting novel coronavirus (COVID-19) has become very important owing to the huge global health burden. Till 20 March 2020, a surge of coronavirus disease cases reached over 168 countries and territories around the world with more than 210000 cases including near to 9000 deaths [18]. The increasing worldwide infection of coronavirus disease, it is very important to know it's dynamic as soon and as much as possible. Recently, several mathematical, computational, clinical and examination studies have been put forward for modeling, prediction, treatment and control of the disease, there is still room for improvement. In recent days, modeling novel coronavirus disease has become of extreme importance and several mathematical

Keywords and phrases: Coronavirus disease (COVID-19), mathematical modeling, sensitivity analysis, differential equations, computational simulations.

${ }^{1}$ Departemnt of Mathematics, College of Basic Education, University of Raparin, Ranya, Kurdistant Region of Iraq.

2 Department of Computer Engineering, University of Kurdistan, Sanandaj, Iran.

* Corresponding author: sarbaz.hamza@raparinuni.org

(C) The authors. Published by EDP Sciences, 2020 
and computational researches have been proposed for the prediction of the disease dynamics. Here, we avoid reviewing related preprint papers because of their huge number and lack of formal review evaluation and acceptance by valid journals. One approach proposed by Muhammad and Abdon [5] involved the use of fractional derivative for infection minimization. They considered the seafood market as the main source of infection when the bats and the unknown hosts leave the infection there. After reducing the model into the seafood market, and formulating a fractional model, they parameterized the model using January 2020 data cases. The basic reproduction data calculated by the study was 2.4829 . Sha et al. developed a discrete-time stochastic epidemic model with binomial distributions to study the transmission of the disease [4]. Model parameters are estimated on the basis of fitting to recently reported data from the National Health Commission of the People's Republic of China. Their simulation showed that confirmed cases of the disease will reach the peak near to the end of February 2020 for the country. Tang et al. in [15] constructed a deterministic compartmental model based on the clinical progression of the disease, epidemiological status of the individuals, and intervention measures. Their estimations show that the control reproduction number may be as high as 6.47 . It can be reduced by interventions such as isolation and quarantine, according to a sensitivity analysis. In another study given in [10] that combined a stochastic transmission model with COVID-19 case data from and originated from Wuhan. They modelled transmission as a geometric random walk process, and they used sequential Monte Carlo simulation to infer the transmission rate over time. Based on their calculations, disease transmission probably stopped in Wuhan during late January 2020, but the cases that arrive at international locations from Wuhan, may lead to epidemics eventually. Chen et al. developed a simplified model as Reservoir-People (RP) transmission network and adopted next generation matrix approach to calculate the basic reproduction number to assess the transmissibility of the SARS-CoV-2 [3]. According to their finding, the expected number of secondary infections was 3.58. Tang et al. updated their previous estimation of the transmission risk of the novel coronavirus, based on the recent advances of technological improvement for detection and confirmation of new infected cases [16]. Their calculations were based on a time-dependent dynamic model of contact and diagnose rate, that led to re-estimated daily reproduction number; they observed that the effective daily reproduction ratio has already fallen below 1, end of January 2020. Another mathematical model for this new virus is the efficiency of quarantine measures. The assumption is that all infected individual are isolated after the incubation period. The basic reproduction number $R_{0}$ has an effective role in disease progression [17].

Although numerous modelling methods have been projected so far for new coronavirus disease prediction, a lot can still be improved. Defining models as mass action law with reaction rate constants and calculating the sensitivities for each model state with respect to model parameters, could improve the outcomes. An issue that has not been investigated enough is various local sensitivity analysis for non-normalizations, half normalizations and full normalizations techniques in computational simulations for COVID-19. In a complicated modeling case like new coronavirus dynamics, it is necessary to pay attention to sensitivity analysis more accurately and widely. Even though few related papers used sensitivity analysis in their researches $[12,15]$, the usage was only limited to few special variables and cases. Another novelty of the paper is a simplification of the identification of the critical model parameters, which makes it easy to be used by biologists with less knowledge of mathematical modeling and also facilitates the improvement of the model for future development.

\section{MATHEMATICAL MODELING FOR CORONAVIRUS DisEASE}

There are many models for describing the spread of infectious diseases. The well defined model in this field is "Susceptible-Exposed-Infectious-Recovered" model. This is sometimes called SEIR model [11]. The main idea of the model is based on the clinical progression, epidemiological individuals and intervention measures. Accordingly, the infectious disease SEIR model has been investigated, the model combined with the intervention compartments such as treatment, isolation (hospitalization) and quarantine. We develop the previous model of coronavirus disease (COVID-19) given in $[15,16]$, the model diagram and the interaction individual components given in Figure 1. The model initial populations and interaction parameters are obtained for the confirmed cases in China. 


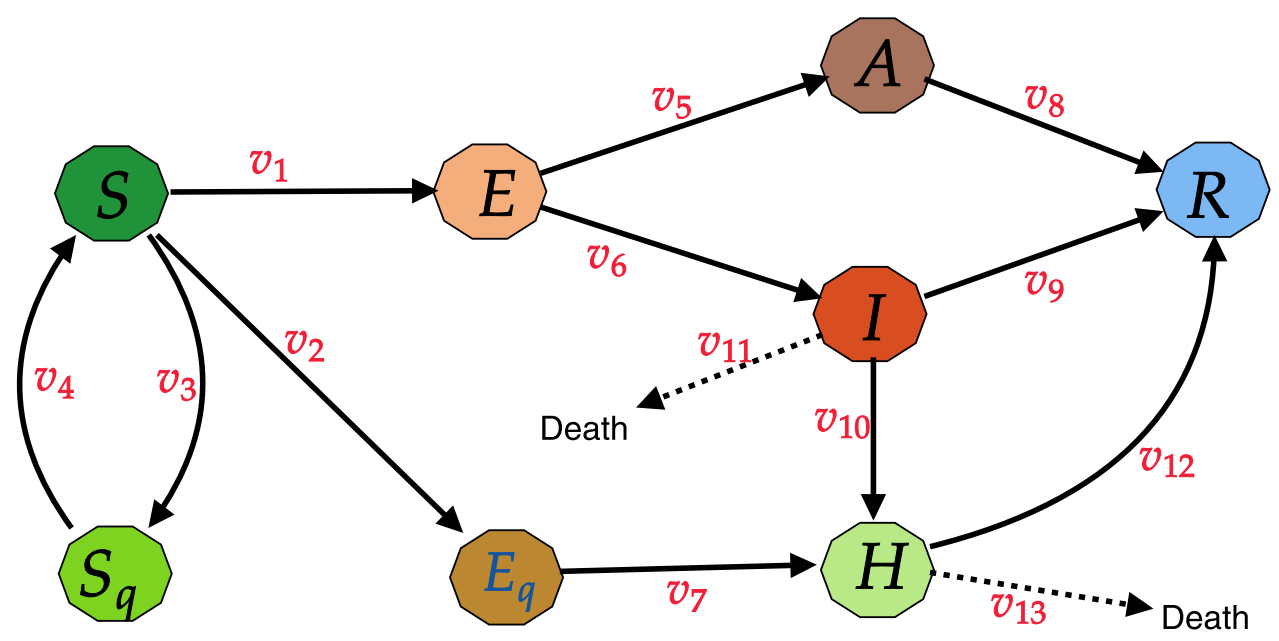

Figure 1. The model interaction individuals for the coronavirus disease (COVID-19) with reaction rates.

The model reactions with their rates are shown below:

$$
\begin{array}{llll}
S \stackrel{v_{1}}{\longrightarrow} E, \quad S \stackrel{v_{2}}{\longrightarrow} E_{q}, & S \stackrel{v_{3}}{\longrightarrow} S_{q} . & S S_{q} \stackrel{v_{4}}{\longrightarrow} S, \\
E \stackrel{v_{5}}{\longrightarrow} A, \quad E \stackrel{v_{6}}{\longrightarrow} I, \quad E_{q} \stackrel{v_{7}}{\longrightarrow} H, & A \stackrel{v_{8}}{\longrightarrow} R, \\
I \stackrel{v_{9}}{\longrightarrow} R, \quad I \stackrel{v_{10}}{\longrightarrow} H, \quad I \stackrel{v_{11}}{\longrightarrow} \text { null, } & H \stackrel{v_{12}}{\longrightarrow} R, \\
H \stackrel{v_{13}}{\longrightarrow} \text { null. }
\end{array}
$$

The model populations (variables) with their biological descriptions are given in Table 1. Furthermore, the model constant interactions (parameters) with their definitions are described in Table 2.

TABLE 1. The model variables for coronavirus disease (COVID-19) with their biological meaning.

\begin{tabular}{ccl}
\hline No. & Variables & Biological Descriptions \\
\hline 1 & $S$ & Susceptible Individuals \\
2 & $E$ & Exposed Individuals \\
3 & $I$ & Symptomatic Infected Individuals \\
4 & $A$ & Pre-symptomatic Individuals \\
5 & $S_{q}$ & Quarantined Susceptible Individuals \\
6 & $E_{q}$ & Quarantined Exposed Individuals \\
7 & $H$ & Quarantined Infected (Hospitalized) Individuals \\
8 & $R$ & Recovered Individuals \\
\hline
\end{tabular}


TABLE 2. The model reaction constants (parameters) and initial individual populations for COVID-19 epidemic outbreak with their biological definitions, all data are confirmed cases in China presented in [15].

\begin{tabular}{|c|c|c|}
\hline Symbols & Biological definitions & Estimated values \\
\hline$S(0)$ & Initial susceptible individuals & $11.081 \times 10^{6}$ \\
\hline$E(0)$ & Initial exposed individuals & 105.1 \\
\hline$I(0)$ & Initial symptomatic infected individuals & 27.679 \\
\hline$A(0)$ & Initial pre-symptomatic infected individuals & 53.839 \\
\hline$S_{q}(0)$ & Initial quarantined susceptible individuals & 739 \\
\hline$E_{q}(0)$ & Initial quarantined exposed individuals & 1.1642 \\
\hline$H(0)$ & Initial quarantined hospitalized individuals & 1 \\
\hline$R(0)$ & Initial recovered individuals & 2 \\
\hline$k_{1}$ & Contact rate & 14.781 \\
\hline$k_{2}$ & Probability of transmission per contact & $2.1011 \times 10^{-8}$ \\
\hline$k_{3}$ & Quarantined rate of exposed individuals & $1.8887 \times 10^{-7}$ \\
\hline$k_{4}$ & Transition rate of exposed individuals to the infected class & $\frac{1}{7}$ \\
\hline$k_{5}$ & The multiple of the transmissibility of $A$ to $I$ & 0.3 \\
\hline$k_{6}$ & $\begin{array}{l}\text { Rate at which the quarantined uninfected contacts were released into the } \\
\text { wider community }\end{array}$ & $\frac{1}{14}$ \\
\hline$k_{7}$ & Probability of having symptoms among infected individuals & 0.86834 \\
\hline$k_{8}$ & $\begin{array}{l}\text { Transition rate of symptomatic infected individuals to the quarantined } \\
\text { infected class }\end{array}$ & 0.13266 \\
\hline$k_{9}$ & $\begin{array}{l}\text { Transition rate of quarantined exposed individuals to the quarantined } \\
\text { infected class }\end{array}$ & 0.1259 \\
\hline$k_{10}$ & Recovery rate of symptomatic infected individuals & 0.33029 \\
\hline$k_{11}$ & Recovery rate of asymptomatic infected individuals & 0.13978 \\
\hline$k_{12}$ & Recovery rate of quarantined infected individuals & 0.11624 \\
\hline$k_{13}$ & Disease-induced death rate & $1.7826 \times 10^{-5}$ \\
\hline
\end{tabular}

TABLE 3. The reaction rates for the model diagram with their definitions.

\begin{tabular}{cl}
\hline Reaction number & Definition of reaction rsates \\
\hline$r_{1}$ & $v_{1}=k_{1} k_{2}\left(1-k_{3}\right) S\left(I+k_{5} A\right)$ \\
$r_{2}$ & $v_{2}=k_{1} k_{2} k_{3} S\left(I+k_{5} A\right)$ \\
$r_{3}$ & $v_{3}=k_{1} k_{3}\left(1-k_{2}\right) S\left(I+k_{5} A\right)$ \\
$r_{4}$ & $v_{4}=k_{6} S_{q}$ \\
$r_{5}$ & $v_{5}=k_{5}\left(1-k_{7}\right) E$ \\
$r_{6}$ & $v_{6}=k_{4} k_{7} E$ \\
$r_{7}$ & $v_{7}=k_{9} E_{q}$ \\
$r_{8}$ & $v_{8}=k_{11} A$ \\
$r_{9}$ & $v_{9}=k_{10} I$ \\
$r_{10}$ & $v_{10}=k_{8} I$ \\
$r_{11}$ & $v_{11}=k_{13} I$ \\
$r_{12}$ & $v_{12}=k_{12} H$ \\
$r_{13}$ & $v_{13}=k_{13} H$ \\
\hline
\end{tabular}


Using equations (2.5)-(2.7), the model dynamics are described by the following system of non-linear ordinary differential equations

$$
\begin{aligned}
& \frac{\mathrm{d} S}{\mathrm{~d} t}=v_{4}-\left(v_{1}+v_{2}+v_{3}\right), \quad \frac{\mathrm{d} E}{\mathrm{~d} t}=v_{1}-\left(v_{5}+v_{6}\right), \\
& \frac{\mathrm{d} I}{\mathrm{~d} t}=v_{6}-\left(v_{9}+v_{10}+v_{11}\right), \quad \frac{\mathrm{d} A}{\mathrm{~d} t}=v_{5}-v_{8}, \\
& \frac{\mathrm{d} S_{q}}{\mathrm{~d} t}=v_{3}-v_{4}, \quad \frac{\mathrm{d} E_{q}}{\mathrm{~d} t}=v_{2}-v_{7}, \\
& \frac{\mathrm{d} H}{\mathrm{~d} t}=v_{7}+v_{10}-\left(v_{12}+v_{13}\right), \quad \frac{\mathrm{d} R}{\mathrm{~d} t}=v_{8}+v_{9}+v_{12} .
\end{aligned}
$$

The model reaction rates $\left\{v_{i}, i=1,2, \ldots, 13\right\}$ are defined in Table 3 .

The model initial populations are expressed in the following equation

$$
\begin{aligned}
& S(0)=S_{0}>0, \quad E(0)=E_{0}>0, \quad I(0)=I_{0}>0, \quad A(0)=A_{0}>0 \\
& S_{q}(0)=S_{q}^{0} \geq 0, \quad E_{q}(0)=E_{q}^{0} \geq 0, \quad H(0)=H_{0} \geq 0, \quad R(0)=R_{0} \geq 0 .
\end{aligned}
$$

In addition, the contacts between susceptible and infected people lead to a "decrease" in the number of susceptible because all model parameters are positive, see Table 2 . We assume $k_{2} \ll 1$ and $k_{3} \ll 1$. These conditions provide " $-S I$ " terms in model (2.2).

\subsection{Sensitivity analysis}

It is necessary for more understanding the biological system dynamics modelled with mass action law to do sensitivity analysis. So, the output of the mathematical model will be investigated easier. Here, we present the basics of our calculations. We consider $n$ reversible reactions which are given below

$$
\sum_{j=1}^{m} a_{i j} x_{j} \underset{k_{i}^{-}}{\stackrel{k_{i}^{+}}{\rightleftharpoons}} \sum_{j=1}^{m} b_{i j} x_{j}, \quad i=1,2, \ldots, n,
$$

where $x_{j}, j=1,2, \ldots, m$ are chemical components, $a_{i j}$ and $b_{i j}$ are non-negative integers. The reaction constants are $k_{i}^{+}>0$ and $k_{i}^{-} \geq 0$. Using the idea of mass action law, the model reaction rates are expressed as follows

$$
v_{i}=k_{i}^{+} \prod_{j=1}^{m} x_{j}^{a_{i j}}(t)-k_{i}^{-} \prod_{j=1}^{m} x_{j}^{b_{i j}}(t) .
$$

Therefore, the model mathematical equations are defined below

$$
\frac{\mathrm{d} x}{\mathrm{~d} t}=\sum_{i \in J \subset \mathbb{R}} \pi_{i} v_{i},
$$

where $\pi_{i j}=b_{i j}-a_{i j}$, for $i=1,2, \ldots, n$ and $j=1,2, . ., m$. Equation (2.6) can be written as follows:

$$
\frac{\mathrm{d} x_{j}}{\mathrm{~d} t}=h_{j}(x, k)
$$


where $x \in \mathbb{R}^{m}$ and $k \in \mathbb{R}^{n}$. The more details and descriptions about chemical reactions and their differential equations with some applications in system biology can be found in [6]. An assumption is that the model input is a vector of parameters, and the model output is a vector of model states(variables). Local sensitivity is the change in model variables $x_{j}, j=1,2, . ., m$ with respect to the model parameters $k_{p}, p=1,2, \ldots, n$. Basically, the model equation of sensitivity for each variable with respect to the parameters is given

$$
s_{j p}=\frac{\partial x_{j}}{\partial k_{p}}=\lim _{\Delta k_{p} \rightarrow 0} \frac{x_{j}\left(k_{p}+\Delta k_{p}\right)-x_{j}\left(k_{p}\right)}{\Delta k_{p}} .
$$

Finite difference approximation can be used to calculate equation (2.8) as follows:

$$
s_{j p}=\frac{\partial x_{j}}{\partial k_{p}} \approx \frac{x_{j}\left(k_{p}+\Delta k_{p}\right)-x_{j}\left(k_{p}\right)}{\Delta k_{p}} .
$$

Generally, direct sensitivity analysis has been used to calculate sensitivity equation that differential equations are solved for sensitivity coefficients.

$$
\frac{\partial s_{j p}}{\partial t}=\frac{\partial}{\partial t}\left(\frac{\partial x_{j}}{\partial k_{p}}\right)=\frac{\partial}{\partial k_{p}}\left(\frac{\partial x_{j}}{\partial t}\right)=\frac{\partial}{\partial k_{p}}\left(h_{j}(x(t), k) .\right.
$$

Equation (2.10) can be further driven by the chain rule of differentiation. Finally, the local sensitivity equation takes the Jacobian matrix as follows

$$
\dot{\mathcal{S}}=\mathcal{H}_{k_{p}}+\mathcal{J} \mathcal{S}, \quad p=1,2, \ldots, n,
$$

where the matrices $\mathcal{S}, \mathcal{H}_{k_{p}}$ and $\mathcal{J}$ are defined by

$$
\mathcal{S}=\left(\begin{array}{c}
\frac{\partial x_{1}}{\partial k_{p}} \\
\frac{\partial x_{2}}{\partial k_{p}} \\
\vdots \\
\frac{\partial x_{m}}{\partial k_{p}}
\end{array}\right), \quad \mathcal{H}_{k_{p}}=\left(\begin{array}{c}
\frac{\partial h_{1}}{\partial k_{p}} \\
\frac{\partial h_{2}}{\partial k_{p}} \\
\vdots \\
\frac{\partial h_{m}}{\partial k_{p}}
\end{array}\right), \quad \mathcal{J}=\left(\begin{array}{cccc}
\frac{\partial h_{1}}{\partial x_{1}} & \frac{\partial h_{1}}{\partial x_{2}} & \cdots & \frac{\partial h_{1}}{\partial x_{m}} \\
\frac{\partial h_{2}}{\partial x_{1}} & \frac{\partial h_{2}}{\partial x_{2}} & \cdots & \frac{\partial h_{2}}{\partial x_{m}} \\
\vdots & \vdots & \ddots & \vdots \\
\frac{\partial h_{m}}{\partial x_{1}} & \frac{\partial h_{m}}{\partial x_{2}} & \cdots & \frac{\partial h_{m}}{\partial x_{m}}
\end{array}\right)
$$

The initial conditions of system (2.11) are determined by the input parameter $k_{p}$ and the initial conditions of the output variables $x_{j}$. Readers can see further details and improvements of sensitivity analysis with its applications on systems biology in $[1,2,6-9,13,14,19]$. The local sensitivity values given in equation $(2.11)$ can be computed using SimBiology Toolbox in MATLAB with three different techniques: non normalizations, half normalizations and full normalizations. Accordingly, in a complicated modeling case like new coronavirus dynamics, it is necessary to pay attention to sensitivity analysis more accurately and widely. Thus, we analyze the coronavirus equations given in system (2.2) and calculating all local sensitivities for the model states with respect to model parameters.

\section{Computational Results}

The complex models of the spread of infectious diseases sometimes can not be well understood only by biological tools. This is why computational simulations and mathematical modeling is used, specially in helping health care programs. Studying these models theoretically helps biologists to predict future model dynamics and identify critical model parameters. The values of parameters and initial populations in this study are obtained 


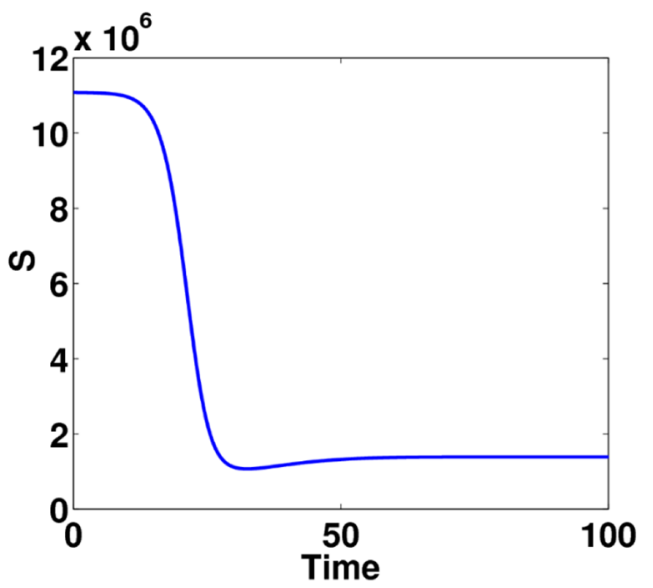

(a)

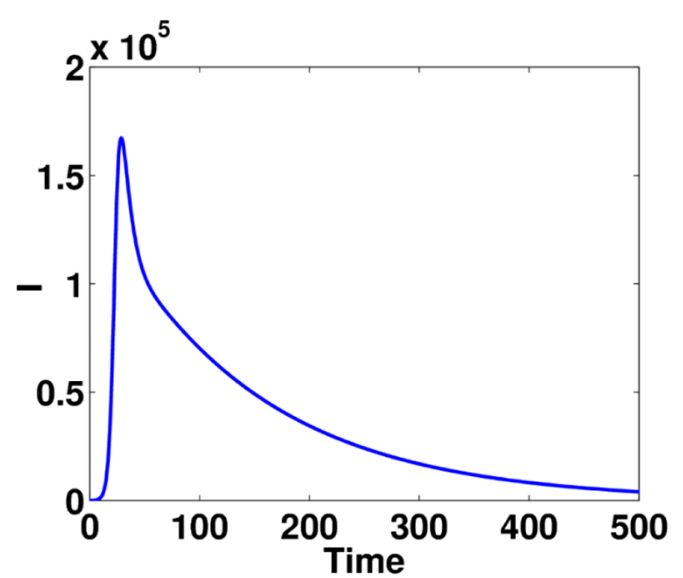

(c)

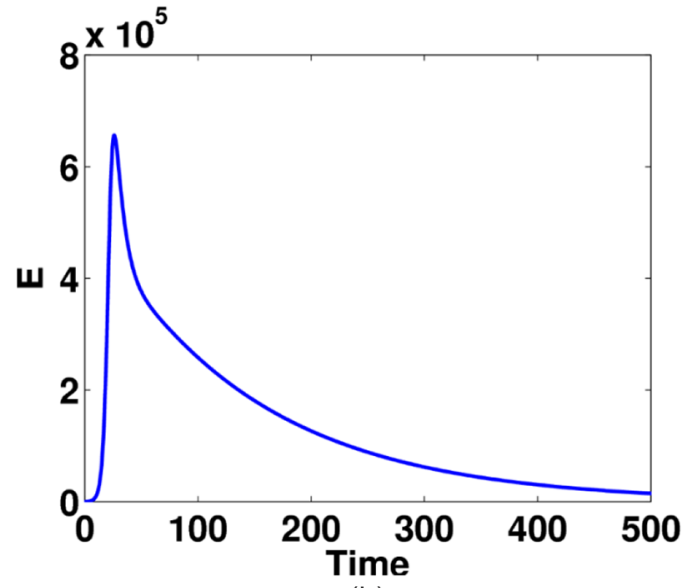

(b)

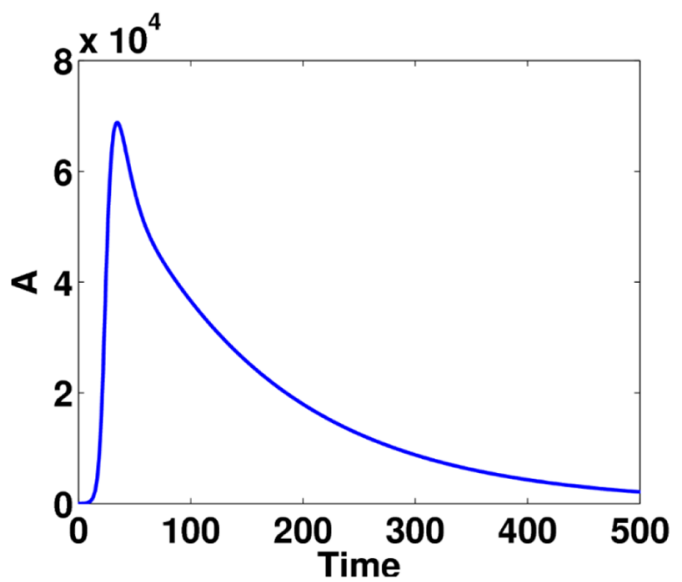

(d)

Figure 2. Computational simulations for the model states given in system (2.2) of the COVID19 using MATLAB, vertical lines represent model populations and horizontal lines show time in days, there are model dynamics of (a) susceptible individuals, (b) exposed individuals (c) symptomatic infected individuals, (d) pre-symptomatic infected individuals.

from the WHO situation report (the National Health Commission of the Republic of China ) presented in [15]. We calculate the numerical approximate solutions of the model equations (2.2) for different parameters and initial populations using SBedit for MATLAB; see Figures 2 and 3. Accordingly, this concludes that there is a different model dynamic for population model states. Numerical simulations are computed in two dimensional planes for model parameters and initial populations obtained from the WHO situation report given in [15]. Results in this study provide a good step forward in predicting the model dynamics in the future for development programs, interventions and health care strategies.

Another forward step for further study and model development is using the idea of local sensitivity analysis presented in equation (2.11). We use SimBiology Toolbox for MATLAB to calculate the local sensitivity of each model state with respect to model constants. We compute the model sensitivities using three different techniques: non normalizations, half normalizations and full normalizations; see Figures 4-6. Interestingly, results provide us further understanding to the model and give ones to identify the critical model parameters. For example, it seems that the set $\left\{k_{2}, k_{5}, k_{6}, k_{7}, k_{8}, k_{10}, k_{13}\right\}$ is the most critical model parameters for the novel coronavirus model while the set $\left\{k_{1}, k_{9}, k_{11}, k_{12}\right\}$ is the less critical model parameters; see Figure 4 . Figure 5 shows that 


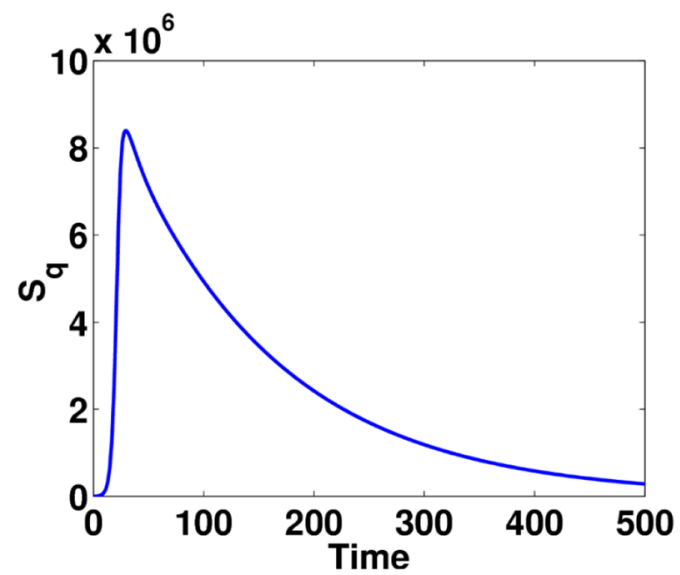

(a)

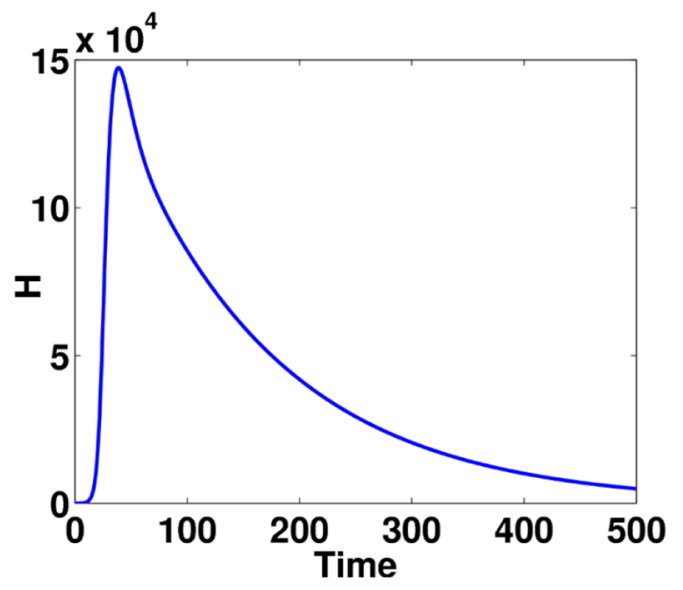

(c)

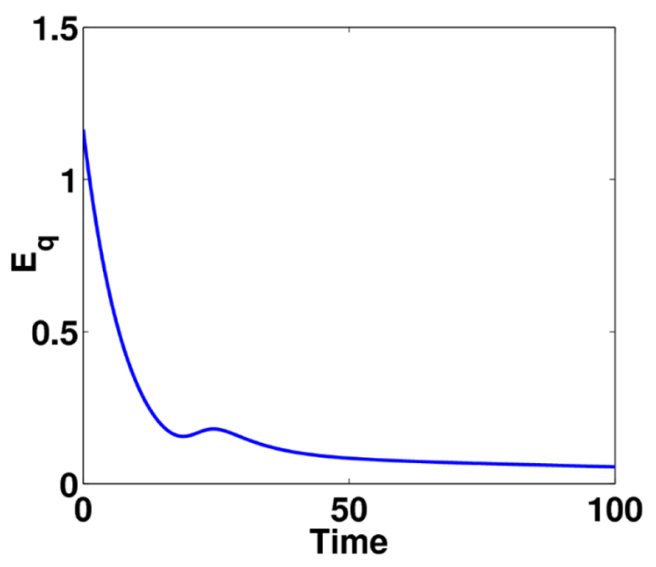

(b)

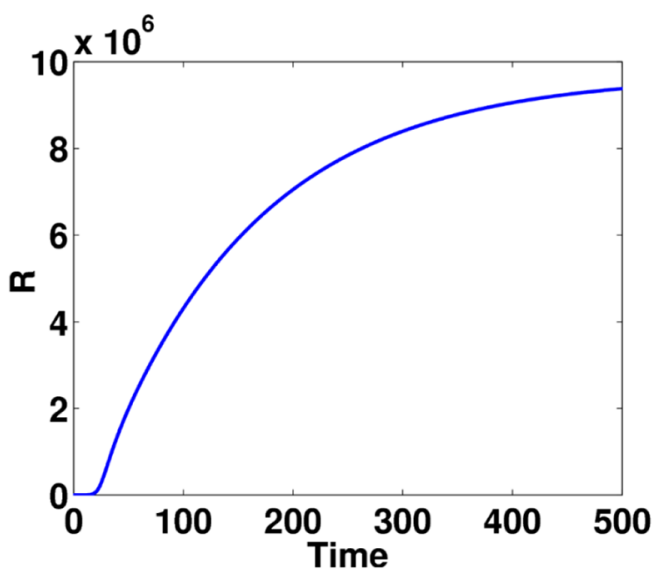

(d)

FIgure 3. Computational simulations for the model states given in system (2.2) of the COVID19 using MATLAB, vertical lines represent model populations and horizontal lines show time in days, there are model dynamics of (a) quarantined susceptible individuals, (b) quarantined exposed individuals (c) quarantined hospitalized individuals, (d) recovered individuals.

parameters $k_{1}, k_{6}, k_{9}$ and $k_{12}$ are mainly the less critical model parameters while the other model parameters are generally sensitive to the model. Figure 6 provides us that the set of parameters $\left\{k_{6}, k_{9}, k_{12}, k_{13}\right\}$ is the less sensitive model parameters, whereas the rest of the model parameters become sensitive for the model variables. As a result, identifying critical model parameters in this study based on computational simulations is an effective way to further study the model practically and theoretically and give some suggestions for future improvements of the novel coronavirus vaccination programs, interventions and controlling the spread of disease.

\section{Discussions}

Global efforts around the world are focused and discussed several healthcare strategies for minimizing the impact of the new coronavirus on community. As it is clear, this virus becomes a public health threat and spreading easily among individuals. Results in this study suggested that health care programs should more pay attention on the set of sensitive parameters compared to less sensitive parameters. This is to reduce the number of infected individuals from the disease and to prevent the new conronavirus propagation more widely on the 


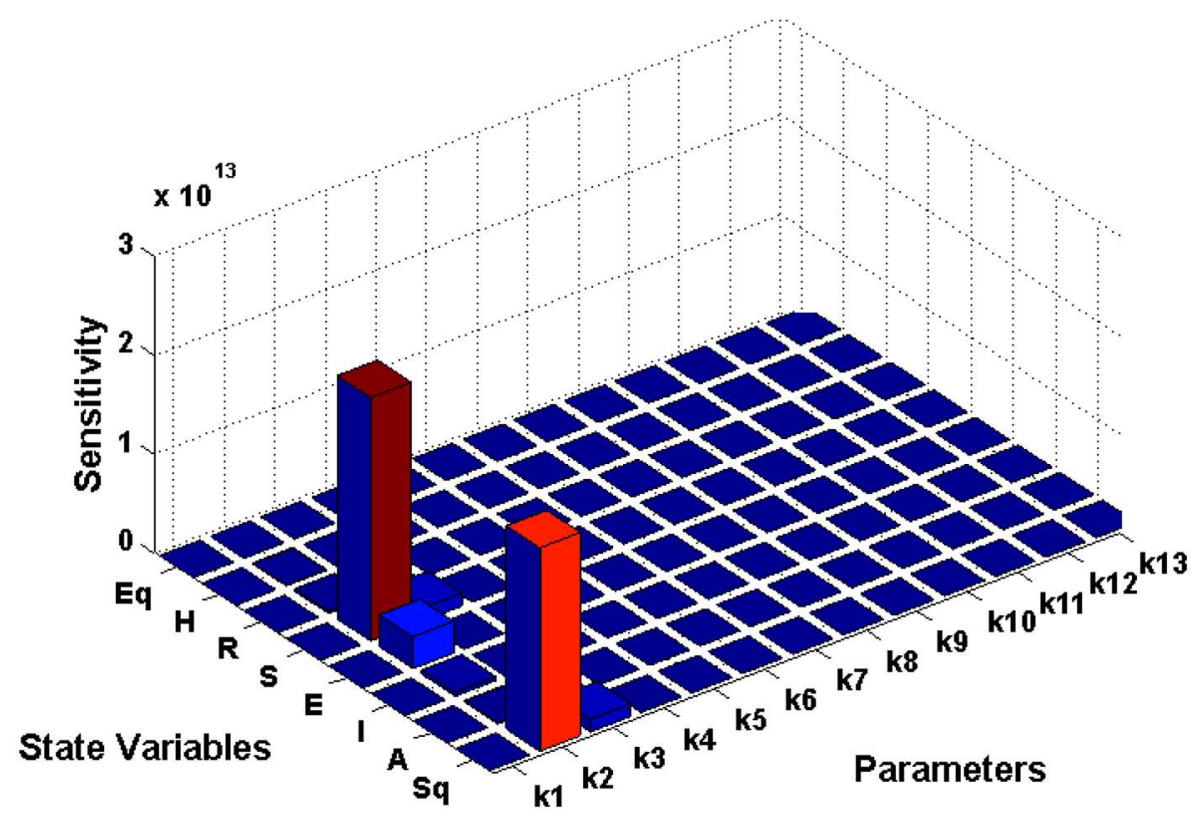

(a)

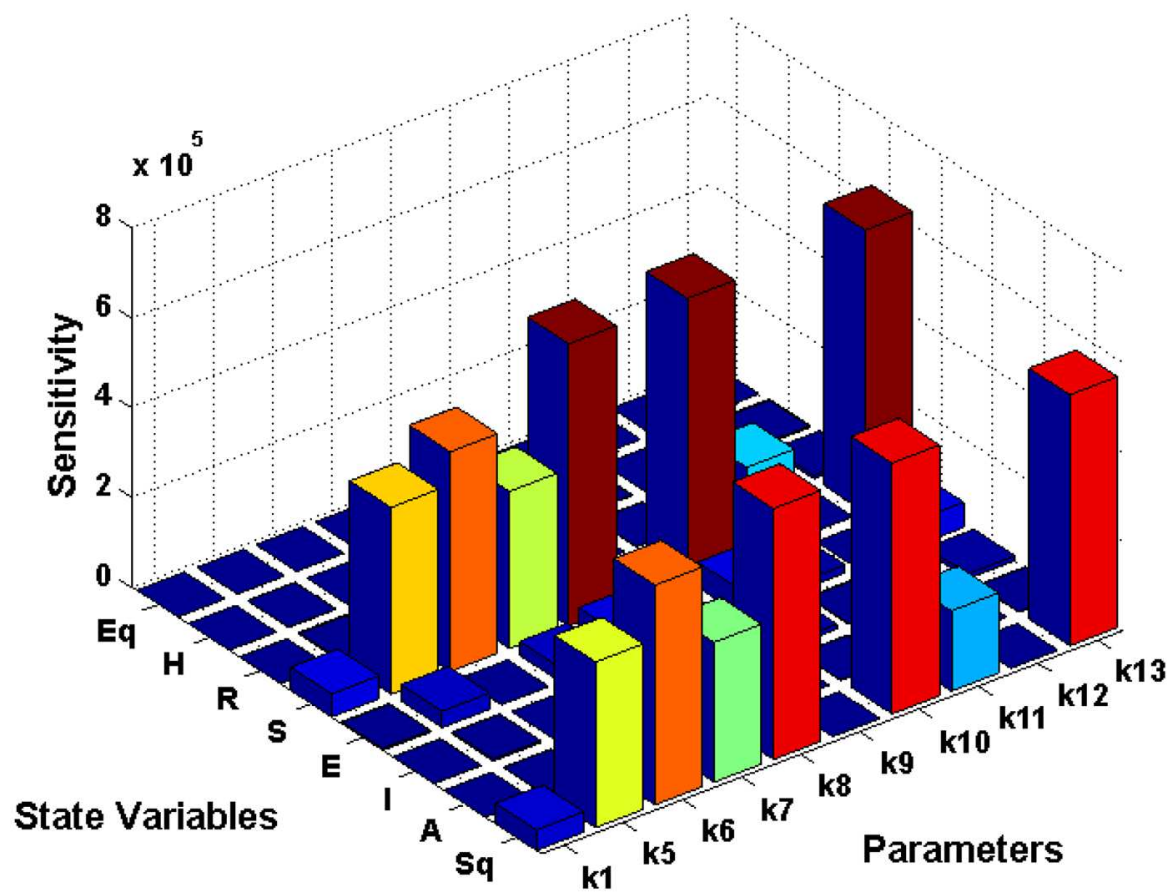

(b)

FiguRE 4. Local sensitivity analysis with non-normalizations in computational simulations for the coronavirus disease (COVID-19) using MATLAB, in this technique the parameters $k_{2}, k_{3}$ and $k_{4}$ are very sensitive compared to the other parameters thus two figures are computed, (a) the sensitivity of all variables with respect to all parameters, (b) the sensitivity of all variables with respect to all parameters except $k_{2}, k_{3}$ and $k_{4}$. 


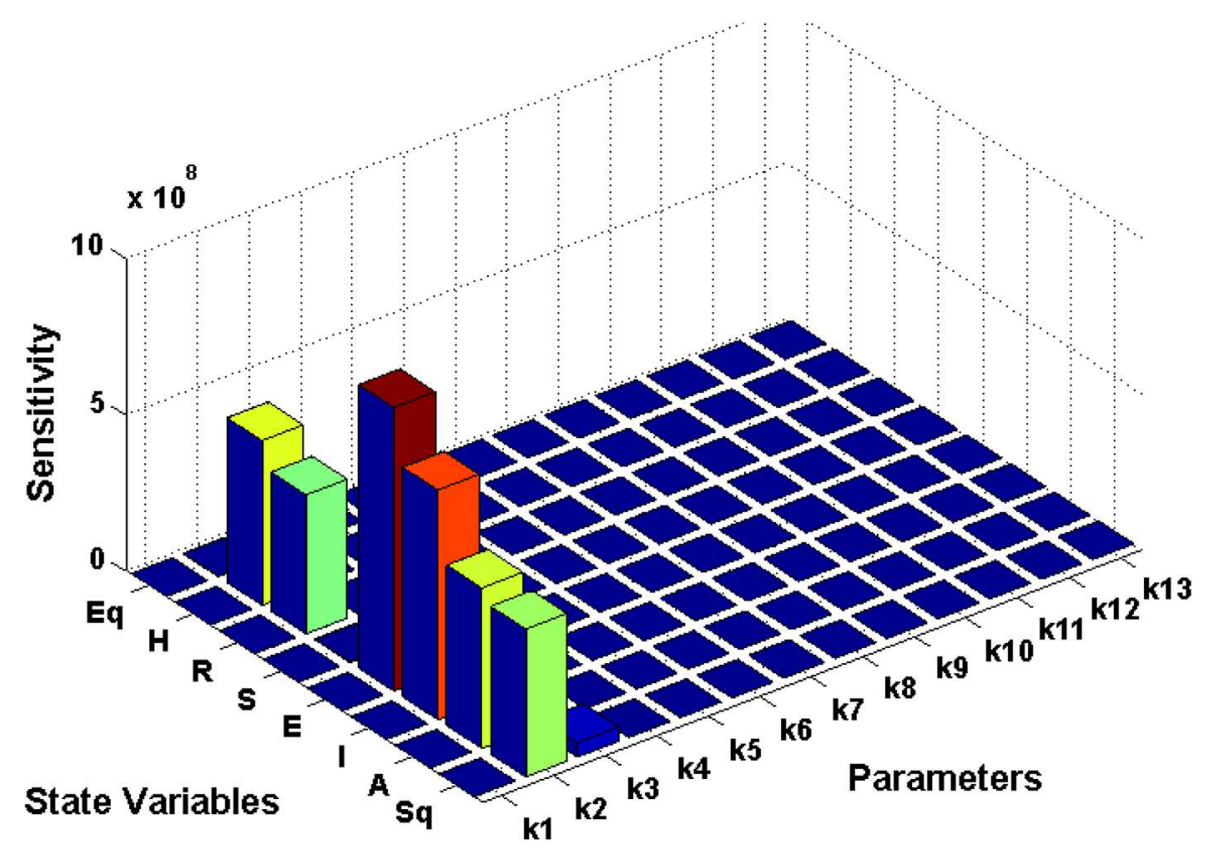

(a)

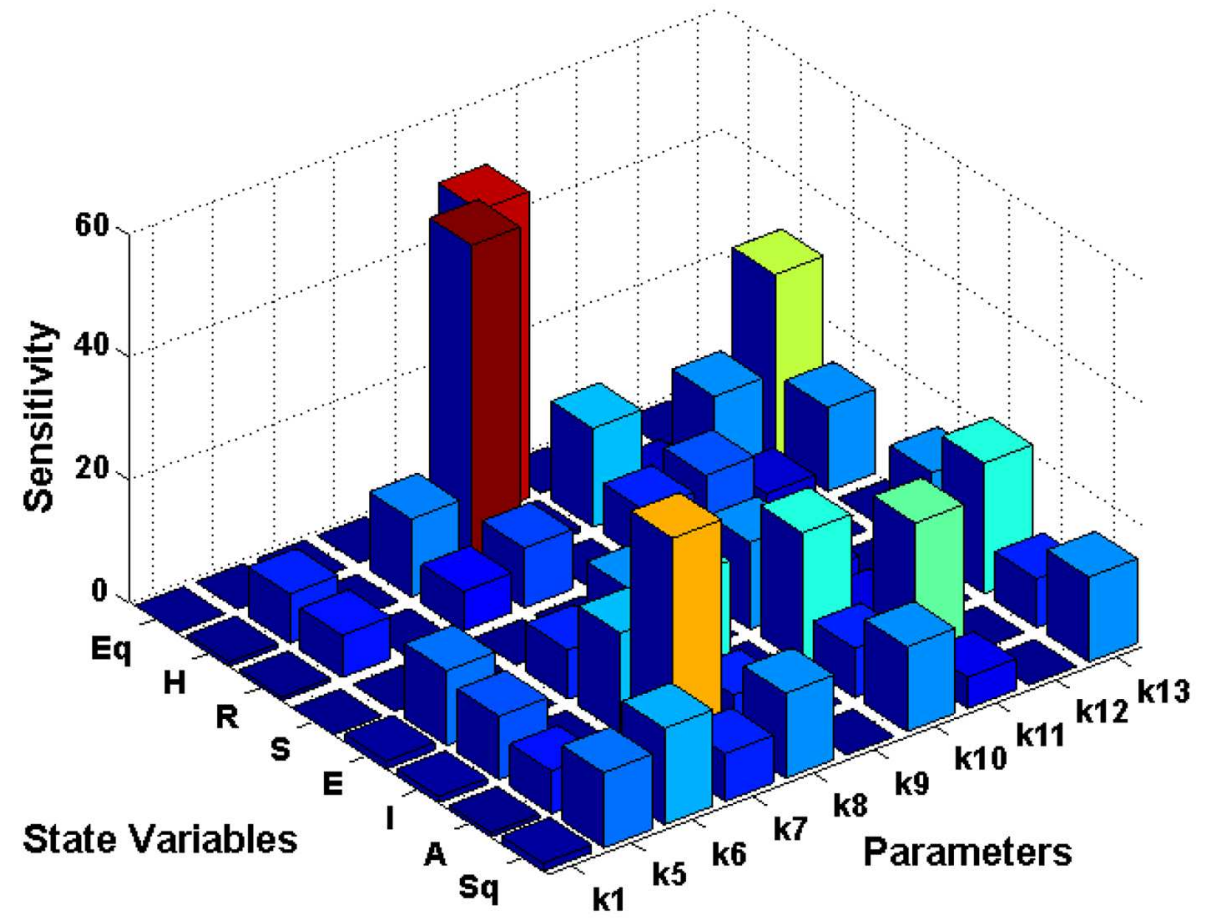

(b)

FIGURE 5. Local sensitivity analysis with half normalizations in computational simulations for the coronavirus disease (COVID-19) using MATLAB, in this technique the parameters $k_{2}, k_{3}$ and $k_{4}$ are very sensitive compared to the other parameters thus two figures are computed, (a) the sensitivity of all variables with respect to all parameters, (b) the sensitivity of all variables with respect to all parameters except $k_{2}, k_{3}$ and $k_{4}$. 


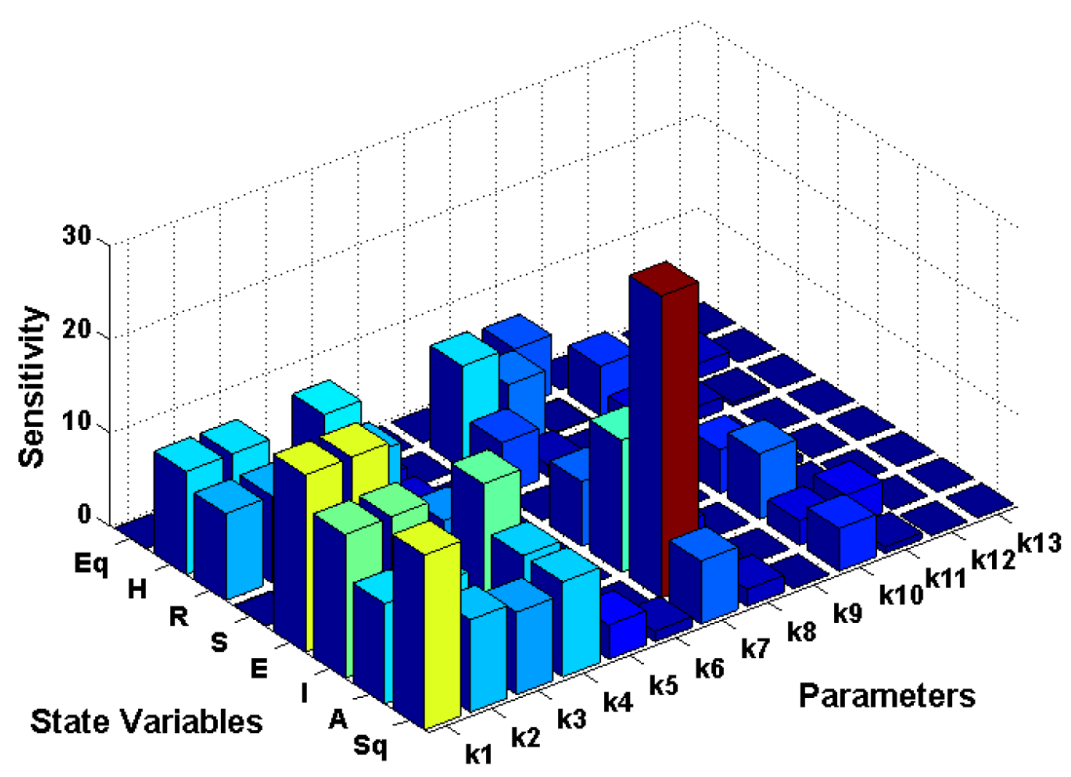

(a)

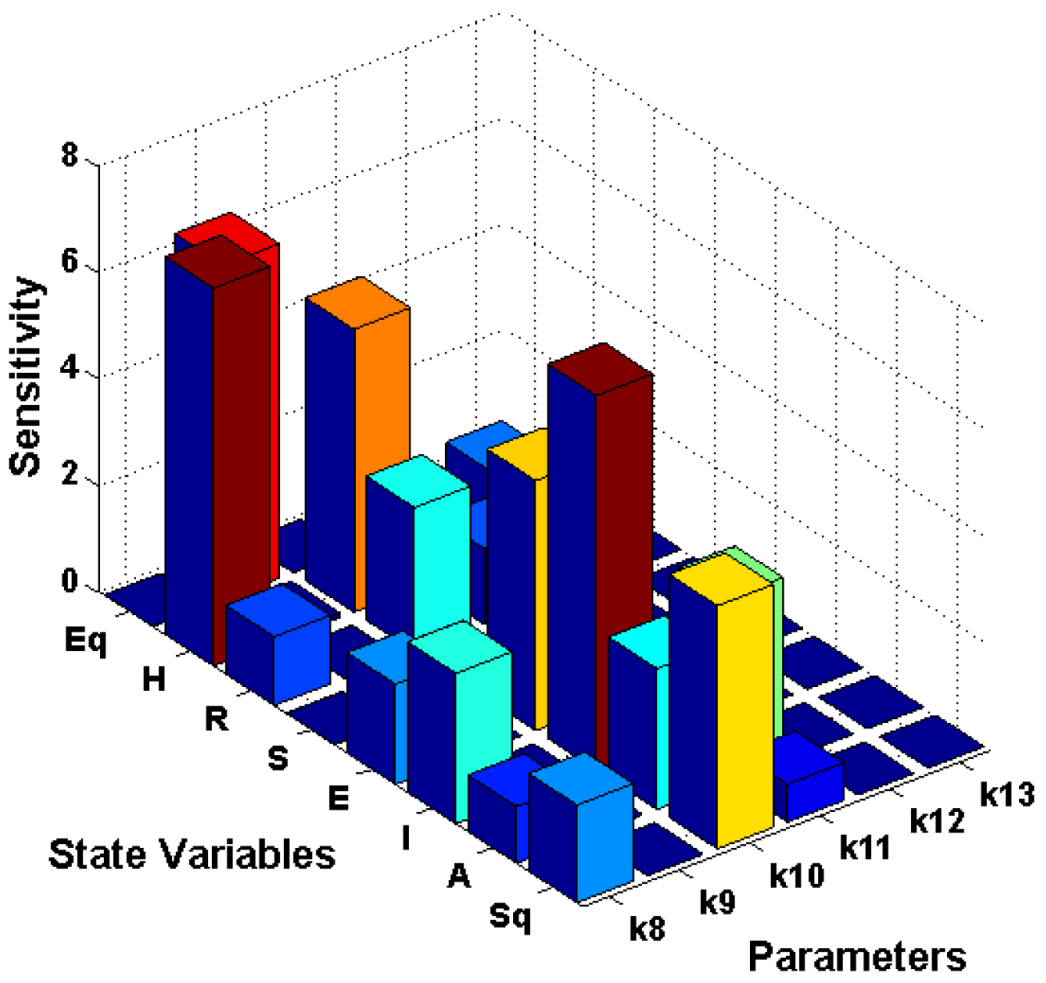

(b)

FigURE 6. Local sensitivity analysis with full normalizations in computational simulations for the coronavirus disease (COVID-19) using MATLAB, in this technique the set of parameters $\left\{k_{1}, k_{2}, k_{3}, k_{4}, k_{5}, k_{6}, k_{7}\right\}$ are very sensitive compared to the other parameters thus two figures are computed, (a) the sensitivity of all variables with respect to all parameters, (b) the sensitivity of all variables with respect to the set of parameters $\left\{k_{8}, k_{9}, k_{10}, k_{11}, k_{12}, k_{13}\right\}$. 
community. It may help community interventions that can mitigate the impact of coronavirus disease. Computational results here provide some important suggestions to healthcare responsibilities as the most important response strategy to delay the spread of disease.

Interestingly, calculating sensitivity analysis based on non-normalization technique shows some important points. Firstly, susceptible $(S)$, exposed $(E)$ and quarantined susceptible $\left(S_{q}\right)$ individuals are very sensitive to $k_{2}$ (transmission per contact); see Figure 4a. Another interesting result is that almost all model parameters may have an affective role on spreading this virus among susceptible and quarantined susceptible people; see Figure $4 \mathrm{~b}$. This gives us how public health partners pay more attention priority on interventions for these two groups. According to local sensitivity analysis with half normalizations in computational simulations, a set of individuals $E, I, A, S_{q}, H, R$ are very sensitive to the parameter $k_{2}$; see Figure 5 a. This is again contact between person-to-person has a great role in spreading this disease. The other factors may have also role to infect people in different levels, this is clearly occurred in our computational simulation given in Figure 5b. Specially, transition rate of symptomatic infected individuals to the quarantined infected class, impacts on the hospital capacity. Accordingly, computational results based on local sensitivity for full normalizations show that most of the reaction rate constants have a great role on model variable sensitivities, this clearly shown in Figure 6. More interestingly, it can be concluded that the most effective factors on spreading coronavirus are person-to-person communicate rate, quarantined exposed rate and transition rate of exposed individuals to the infected individuals. Our computational results confirms that healthcare responsible organizations in the worldwide should more pay attention to stay people at home and reduce the communication and contacts.

Similar to the findings of Figure 5b, Figure $6 \mathrm{~b}$ demonstrates that $\mathrm{H}$, the number of hospitalized patients, is very sensitive to $k_{8}$ (transition rate of symptomatic infected individuals to the quarantined infected class). Moreover, it can be concluded from the Figure 6a that the more the quarantined rate of exposed individuals controlled, the less the susceptible individuals, such as old persons, need to be quarantined. Accordingly, it can be seen that the quarantined exposed individuals $E_{q}$ are more sensitive to $k_{9}$ (transition rate of quarantined exposed individuals to the quarantined infected class), see Figures $5 \mathrm{~b}$ and $6 \mathrm{~b}$. It can be suggested that their movements should be limited outside of their home or current places. In the quarantine places, anyone there should separate themselves from others by staying in a specific space and using a different equipment, bathroom and kitchen.

\section{Conclusions}

Studying and identifying critical model parameters for the infectious disease models are difficult tasks. Describing the model dynamics for such systems needs some mathematical methods. However computational simulations and sensitivity analysis will help further understudying and future prediction about model dynamics. The coronavirus disease (COVID-19) model is a complicated one, and it requires some mathematical tools to have improvements about interventions and healthcare programs. In this study, we reviewed some previous research about this disease and we suggested the mathematical modeling for further research and developments. The dynamics of model states are discussed with some computational simulations.

We applied the idea of local sensitivity to calculate the sensitivity of each model state with respect to model parameters. Three different techniques are investigated to calculate the model sensitivities which are non normalizations, half normalizations and full normalizations. These provide us an important step forward to identify model critical elements. Computational results may help international efforts to reduce number of infected individuals from the disease and to prevent the propagation of new conronavirus more widely on the community. For example, the most effective factors on spreading coronavirus based on our results are communicate rate person-to-person, quarantined exposed rate and transition rate of exposed individuals to the infected individuals.

Furthermore, regard to the potential lacking of hospital capacity and its strong effect on the national government healthcare responsible efficacy around the world. It seems necessary to plan a certain strategy to prevent the hospital beds crisis, such as putting the susceptible individuals on quarantine sooner rather than later. Accordingly, the healthcare communities should pay more attention in the quarantine places for controlling this 
disease more effectively. It can be strongly suggested that anyone in the quarantine places should be separated from the others and should use only their own equipment, bedroom and toilet to prevent the transmission of the virus through the touching of shared surfaces. Interestingly, the proposed steps here can further be developed and applied to a wide range of coronavirus models for different cases around the world. It will be useful for future model improvements, interventions and vaccination programs.

Acknowledgements. This research was partially supported by the University of Raparin. We are thankful to our universities for providing us with wonderful facilities. These supports are greatly appreciated.

\section{DECLARATION OF INTEREST}

The authors declare that there are no competing interests.

\section{REFERENCES}

[1] A. Akgül, S.H.A. Khoshnaw and W.H. Mohammed, Mathematical Model for the Ebola Virus Disease. J. Adv. Phys. 7 (2018) 190-198.

[2] A.C. Babtie, P. Kirk and M.P.H. Stumpf, Topological sensitivity analysis for systems biology. Proc. Natl. Acad. Sci. 111 (2014) 18507-18512.

[3] T.-M. Chen, J. Rui, Q.-P. Wang, Z.-Y. Zhao, J.-A. Cui and L. Yin, A mathematical model for simulating the phase-based transmissibility of a novel coronavirus. Infect. Dis. Poverty 9 (2020) 1-8.

[4] S. He, S. Tang and L. Rong, A discrete stochastic model of the COVID-19 outbreak: Forecast and control. Math. Biosci. Eng. $17(2020) 13$.

[5] M.A. Khan and A. Atangana, Modeling the dynamics of novel coronavirus (2019-nCov) with fractional derivative. To appear in: Alex. Eng. J. https://doi.org/10.1016/j.aej.2020.02.033 (2020).

[6] S.H.A. Khoshnaw, Model reductions in biochemical reaction networks. Ph.D. thesis, University Leicester-UK (2015).

[7] S.H.A. Khoshnaw, A mathematical modelling approach for childhood vaccination with some computational simulations. AIP Conf. Proc. 2096 (2019) 020022.

[8] S.H.A. Khoshnaw, N.A. Mohammad and R.H. Salih, Identifying critical parameters in SIR model for spread of disease. Open J. Model. Simul. 5 (2017) 32-46.

[9] A. Kiparissides, S.S. Kucherenko, A. Mantalaris and E.N. Pistikopoulos, Global sensitivity analysis challenges in biological systems modeling. Ind. Eng. Chem. Res. 48 (2009) 7168-7180.

[10] A.J. Kucharski, T.W. Russell, C. Diamond, Y. Liu, J. Edmunds, S. Funk and J.D. Munday, Early dynamics of transmission and control of COVID-19: a mathematical modelling study. Lancet Infect. Dis. 20 (2020) 553.

[11] M.Y. Li, J.R. Graef, L. Wang and J. Karsai, Global dynamics of a SEIR model with varying total population size. Math. Biosci. 160 (1999) 191-213.

[12] L. Li, T. Huang, Y. Wang, Z. Wang, Y. Liang, T. Huang and Y. Wang, 2019 novel coronavirus patients' clinical characteristics, discharge rate and fatality rate of meta-analysis. J. Med. Virol. 96 (2020) 577-583.

[13] D.R. Powell, J. Fair, R.J. LeClaire, L.M. Moore and D. Thompson, Sensitivity analysis of an infectious disease model, in Proceedings of the International System Dynamics Conference (2005).

[14] H. Rabitz, M. Kramer and D. Dacol, Sensitivity analysis in chemical kinetics. Ann. Rev. Phys. Chem. 34 (1983) $419-461$.

[15] B. Tang, X. Wang, Q. Li, N.L. Bragazzi, S. Tang, Y. Xiao and J. Wu, Estimation of the transmission risk of the 2019-nCoV and its implication for public health interventions. J. Clin. Med. 9 (2020) 462.

[16] B. Tang, N.L. Bragazzi, Q. Li, S. Tang, Y. Xiao and J. Wu, An updated estimation of the risk of transmission of the novel coronavirus (2019-nCov). Infect. Dis. Model. 5 (2020) 248-255.

[17] V. Volpert, M. Banerjee and S. Petrovskii, On a quarantine model of coronavirus infection and data analysis. MMNP $\mathbf{1 5}$ (2020) 24.

[18] WHO, Novel coronavirus (COVID-19) situation. Available from https://experience.arcgis.com/experience/ 685d0ace521648f8a5beeee1b9125cd (2020).

[19] Z. Zi, Sensitivity analysis approaches applied to systems biology models. IET Syst. Biol. 5 (2011) 336-346. 